Acta Crystallographica Section E

Structure Reports

Online

ISSN 1600-5368

\section{Hazel S. Wilkinson and William T. A. Harrison*}

Department of Chemistry, University of Aberdeen, Meston Walk, Aberdeen AB24 3UE, Scotland

Correspondence e-mail:

w.harrison@abdn.ac.uk

\section{Key indicators}

Single-crystal X-ray study

$T=295 \mathrm{~K}$

Mean $\sigma(\mathrm{s}-\mathrm{O})=0.001 \AA$

$R$ factor $=0.021$

$w R$ factor $=0.058$

Data-to-parameter ratio $=28.3$

For details of how these key indicators were automatically derived from the article, see http://journals.iucr.org/e.

\title{
Guanidinium dihydrogenarsenate
}

The title compound, $\left(\mathrm{CH}_{6} \mathrm{~N}_{3}\right)\left[\mathrm{H}_{2} \mathrm{AsO}_{4}\right]$, contains a network of guanidinium cations and dihydrogenarsenate anions. The component species interact by way of cation-to-anion $\mathrm{N}-$ $\mathrm{H} \cdots \mathrm{O}$ and anion-to-anion $\mathrm{O}-\mathrm{H} \cdots \mathrm{O}$ hydrogen bonds, the latter leading to infinite sheets of $\left[\mathrm{H}_{2} \mathrm{AsO}_{4}\right]^{-}$anions.

\section{Comment}

The title compound, (I), was prepared as part of ongoing studies of hydrogen-bonding interactions in molecular salts (Wilkinson \& Harrison, 2004).<smiles>NC(N)=[NH2+]</smiles>

The $\left[\mathrm{H}_{2} \mathrm{AsO}_{4}\right]^{-}$anion in (I) shows its normal tetrahedral geometry (Fig. 1) about As [mean As-O = 1.684 (2) $\AA$ ], with the usual distinction between protonated and unprotonated As-O bond lengths (Wilkinson \& Harrison, 2004); see Table 1. The three $\mathrm{C}-\mathrm{N}$ bond lengths in the propeller-shaped $\left(\mathrm{CH}_{6} \mathrm{~N}_{3}\right)^{+}$cation are similar (Table 1), indicating that the usual model of electron delocalization in this species, leading to a $\mathrm{C}-\mathrm{N}$ bond order of 1.33, is applicable here.

As well as Coulombic forces, the component species in (I) interact by means of a network of cation-to-anion $\mathrm{N}-\mathrm{H} \cdots \mathrm{O}$ and anion-to-anion $\mathrm{O}-\mathrm{H} \cdots \mathrm{O}$ hydrogen bonds, as detailed in Table 2. All the guanidinium atoms participate in hydrogen bonds (one of which, via $\mathrm{H} 8$, is bifurcated with notably longer $\mathrm{H} \cdots \mathrm{O}$ separations than the others), such that the $\left(\mathrm{CH}_{6} \mathrm{~N}_{3}\right)^{+}$ cation makes $\mathrm{N}-\mathrm{H} \cdots \mathrm{O}$ links to five adjacent $\left[\mathrm{H}_{2} \mathrm{AsO}_{4}\right]^{-}$ tetrahedra as shown in Fig. 2. For the simple $\mathrm{N}-\mathrm{H} \cdots \mathrm{O}$ bonds, the mean $\mathrm{H} \cdots \mathrm{O}$ distance $=2.18 \AA$, mean $\mathrm{N} \cdots \mathrm{O}=2.951$ (3) $\AA$, and the mean $\mathrm{N}-\mathrm{H} \cdots \mathrm{O}$ angle is $150^{\circ}$.

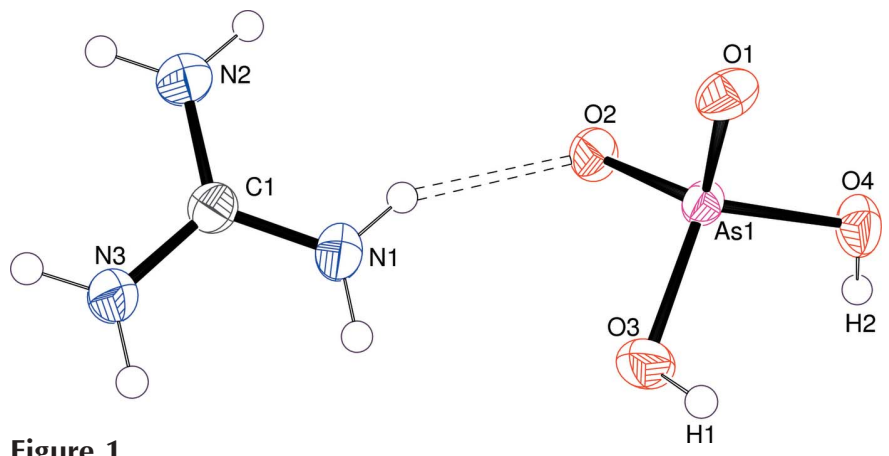

Figure 1

Asymmetric unit of (I) (50\% displacement ellipsoids). The hydrogen bond is indicated by a dashed line.
Received 8 September 2005 Accepted 12 September 2005 Online 17 September 2005
(C) 2005 International Union of Crystallography Printed in Great Britain - all rights reserved 


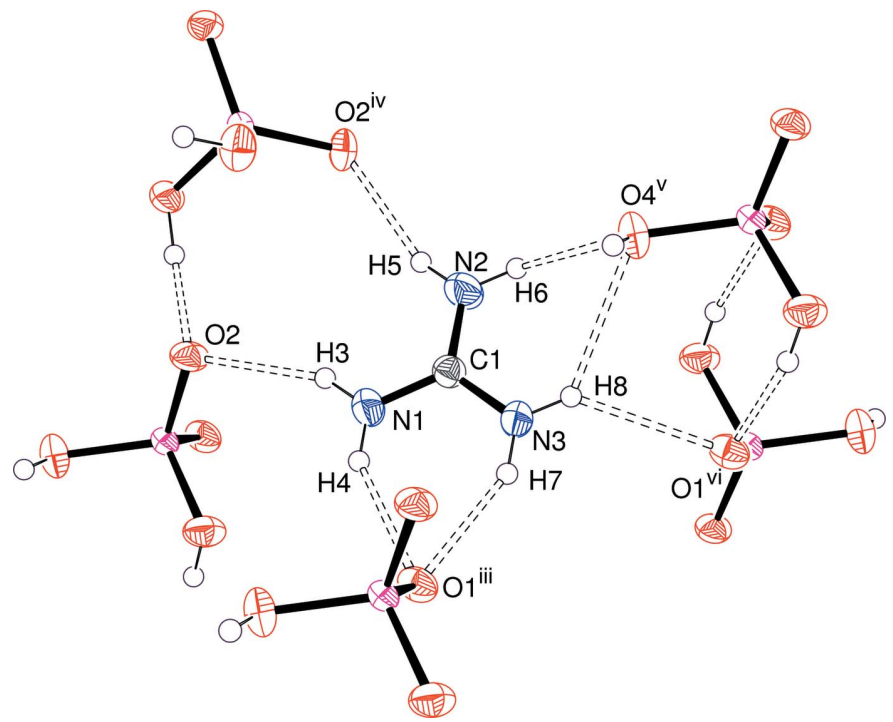

Figure 2

Detail of the cation-to-anion $\mathrm{N}-\mathrm{H} \cdots \mathrm{O}$ links (dashed lines) in (I). Symmetry codes are as in Table 2 .

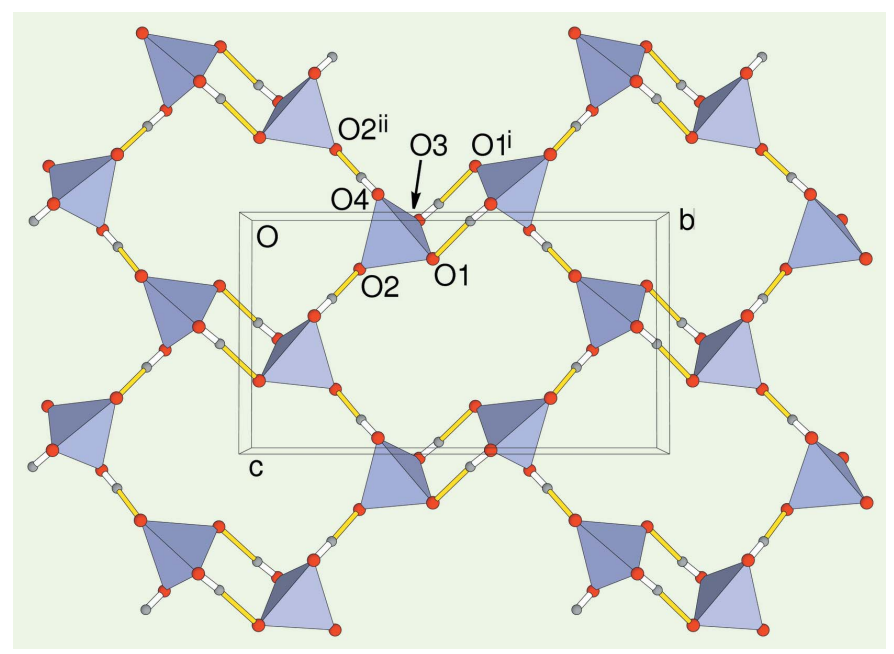

Figure 3

Detail of a part of a (100) hydrogen-bonded sheet of $\left[\mathrm{H}_{2} \mathrm{AsO}_{4}\right]^{-}$groups in (I), in polyhedral representation. Symmetry codes are as in Table 2.

The $\left[\mathrm{H}_{2} \mathrm{AsO}_{4}\right]^{-}$units are linked into infinite sheets (Fig. 3) by way of the $\mathrm{O}-\mathrm{H} \cdots \mathrm{O}$ hydrogen bonds. The $\mathrm{O} 3-\mathrm{H} 1 \cdots \mathrm{O} 1^{\mathrm{i}}$ interaction (see Table 2 for symmetry codes) results in inversion-symmetry-generated dimeric pairs of $\left[\mathrm{H}_{2} \mathrm{AsO}_{4}\right]^{-}$tetrahedra linked by a double (i.e. $\mathrm{O}-\mathrm{H} \cdots \mathrm{O}+\mathrm{O} \cdots \mathrm{H}-\mathrm{O}$ ) hydrogen bond. The $\mathrm{O} 4-\mathrm{H} 2 \cdots \mathrm{O} 2^{\mathrm{ii}}$ bond links the dimers into an infinite sheet (Fig. 3) propagating in (100). The $A s \cdots A s^{i}$ and $A s \cdots A s^{i i}$ separations are $4.0148(3)$ and 5.0190 (3) $\AA$, respectively. If the topological connectivity of the As atoms is considered, a $6^{3}$ sheet $\left(\mathrm{O}^{\prime}\right.$ Keeffe \& Hyde, 1996) arises, i.e. every As node participates in three polyhedral six-ring loops.

The packing for (I) (Fig. 4) results in alternating organic and inorganic layers with respect to the $a$ axis direction. The structure of (I) is distinct from other ammonium hydrogenarsenate salts where isolated pairs of tetrahedra (Todd \&

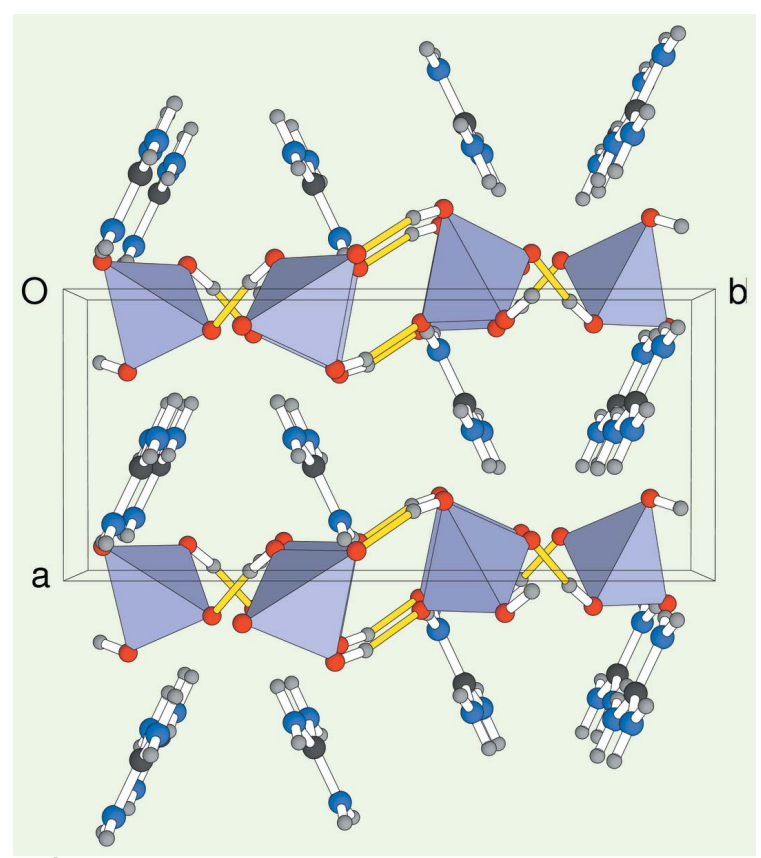

Figure 4

The packing in (I), projected down [001], showing the (100) dihydrogenarsenate layers mediated by guanidinium cations. The $\mathrm{N}-\mathrm{H} \cdots \mathrm{O}$ hydrogen bonds are not shown.

Harrison, 2005) or various kinds of polymeric chains (Wilkinson \& Harrison, 2004) occur.

\section{Experimental}

An aqueous guanidine solution $(0.5 M, 10 \mathrm{ml})$ was added to an $\mathrm{H}_{3} \mathrm{AsO}_{4}$ solution $(0.5 \mathrm{M}, 10 \mathrm{ml})$ to give a clear solution. A mass of chunks and blocks of (I) grew as the water evaporated over the course of a few days.

\section{Crystal data}

$\left(\mathrm{CH}_{6} \mathrm{~N}_{3}\right)\left[\mathrm{H}_{2} \mathrm{AsO}_{4}\right]$

$M_{r}=201.02$

Monoclinic, $P 2_{\mathrm{f}} / c$

$a=6.1571$ (3) А

$b=13.7052(6) \AA$

$c=7.7208$ (3) $\AA$

$\beta=91.715(1)^{\circ}$

$V=651.22(5) \AA^{3}$

$Z=4$

\section{Data collection}

Bruker SMART1000 CCD

diffractometer

$\omega$ scans

Absorption correction: multi-scan

(SADABS; Bruker, 1999)

$T_{\min }=0.172, T_{\max }=0.510$

7302 measured reflections

Refinement

Refinement on $F^{2}$

$R\left[F^{2}>2 \sigma\left(F^{2}\right)\right]=0.021$

$w R\left(F^{2}\right)=0.058$

$S=1.06$

2353 reflections

83 parameters

$\mathrm{H}$-atom parameters constrained

\author{
$D_{x}=2.050 \mathrm{Mg} \mathrm{m}^{-3}$ \\ Mo $K \alpha$ radiation \\ Cell parameters from 4819 \\ reflections \\ $\theta=3.0-32.5^{\circ}$ \\ $\mu=5.18 \mathrm{~mm}^{-1}$ \\ $T=295$ (2) K \\ Block, colourless \\ $0.49 \times 0.29 \times 0.13 \mathrm{~mm}$
}

2353 independent reflections 2068 reflections with $I>2 \sigma(I)$ $R_{\text {int }}=0.027$

$\theta_{\max }=32.5^{\circ}$

$h=-9 \rightarrow 9$

$k=-18 \rightarrow 20$

$l=-11 \rightarrow 11$

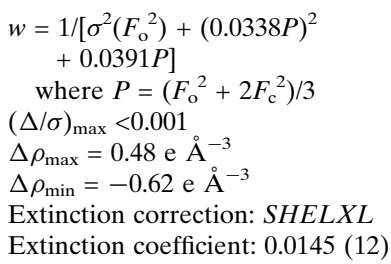


Table 1

Selected bond lengths $(\AA)$.

\begin{tabular}{llll}
\hline As1-O1 & $1.6532(11)$ & $\mathrm{C} 1-\mathrm{N} 1$ & $1.314(2)$ \\
As1-O2 & $1.6538(10)$ & $\mathrm{C} 1-\mathrm{N} 2$ & $1.323(2)$ \\
As1-O4 & $1.7135(10)$ & $\mathrm{C} 1-\mathrm{N} 3$ & $1.324(2)$ \\
As1-O3 & $1.7144(11)$ & & \\
\hline
\end{tabular}

Table 2

Hydrogen-bond geometry $\left(\AA{ }^{\circ}\right)$.

\begin{tabular}{|c|c|c|c|c|}
\hline$D-\mathrm{H} \cdots A$ & $D-\mathrm{H}$ & $\mathrm{H} \cdots A$ & $D \cdots A$ & $D-\mathrm{H} \cdots A$ \\
\hline $\mathrm{O} 3-\mathrm{H} 1 \cdots \mathrm{O} 1^{\mathrm{i}}$ & 0.85 & 1.83 & $2.6684(16)$ & 167 \\
\hline $\mathrm{O} 4-\mathrm{H} 2 \cdots \mathrm{O} 2^{\mathrm{ii}}$ & 0.92 & 1.65 & $2.5648(16)$ & 174 \\
\hline $\mathrm{N} 1-\mathrm{H} 3 \cdots \mathrm{O} 2$ & 0.86 & 2.13 & 2.9185 (18) & 153 \\
\hline $\mathrm{N} 1-\mathrm{H} 4 \cdots \mathrm{O} 1^{\mathrm{iii}}$ & 0.86 & 2.24 & $2.993(2)$ & 147 \\
\hline $\mathrm{N} 2-\mathrm{H} 5 \cdots \mathrm{O} 2^{\mathrm{iv}}$ & 0.86 & 2.15 & $2.955(2)$ & 157 \\
\hline $\mathrm{N} 2-\mathrm{H} 6 \cdots \mathrm{O} 4^{\mathrm{v}}$ & 0.86 & 2.28 & $2.994(2)$ & 140 \\
\hline $\mathrm{N} 3-\mathrm{H} 7 \cdots \mathrm{O} 1^{\mathrm{iii}}$ & 0.86 & 2.11 & $2.8962(17)$ & 152 \\
\hline $\mathrm{N} 3-\mathrm{H} 8 \cdots \mathrm{O} 1^{\mathrm{vi}}$ & 0.86 & 2.47 & $3.1582(18)$ & 138 \\
\hline $\mathrm{N} 3-\mathrm{H} 8 \cdots \mathrm{O} 4^{\mathrm{v}}$ & 0.86 & 2.55 & 3.1965 (18) & 133 \\
\hline
\end{tabular}

Symmetry codes: (i) $-x,-y+1,-z$; (ii) $x,-y+\frac{1}{2}, z-\frac{1}{2}$; (iii) $x+1, y, z$; (iv) $x,-y+\frac{1}{2}, z+\frac{1}{2}$; (v) $x+1, y, z+1$; (vi) $-x+1,-y+1,-z+1$.
The O-bound $\mathrm{H}$ atoms were found in difference maps and allowed for as riding in their as-found relative positions with $U_{\text {iso }}(\mathrm{H})=$ $1.2 U_{\text {eq }}(\mathrm{O})$. The $\mathrm{N}$-bound $\mathrm{H}$ atoms were included in the riding model approximation, with $\mathrm{N}-\mathrm{H}=0.86 \AA$ and with $U_{\text {iso }}(\mathrm{H})=1.2 U_{\text {eq }}(\mathrm{N})$.

Data collection: SMART (Bruker, 1999); cell refinement: SAINT (Bruker, 1999); data reduction: $S A I N T$; $\operatorname{program}(\mathrm{s})$ used to solve structure: SHELXS97 (Sheldrick, 1997); program(s) used to refine structure: SHELXL97 (Sheldrick, 1997); molecular graphics: ORTEP3 (Farrugia, 1997) \& ATOMS (Shape Software, 2004); software used to prepare material for publication: SHELXL97.

HSW thanks the Carnegie Trust for the Universities of Scotland for an undergraduate vacation studentship.

\section{References}

Bruker (1999). SMART (Version 5.624), SAINT (Version 6.02A) and $S A D A B S$. Bruker AXS Inc., Madison, Wisconsin, USA.

Farrugia, L. J. (1997). J. Appl. Cryst. 30, 565.

O'Keeffe, M. \& Hyde, B. G. (1996). Crystal Structures, 1. Patterns and Symmetry, p 357. Washington DC, USA: Mineralogical Society of America. Shape Software (2004). ATOMS. 525 Hidden Valley Road, Kingsport, Tennessee, USA.

Sheldrick, G. M. (1997). SHELXS97 and SHELXL97. University of Göttingen, Germany.

Todd, M. J. \& Harrison, W. T. A. (2005). Acta Cryst. E61, m2026-mm2028. Wilkinson, H. S. \& Harrison, W. T. A. (2004). Acta Cryst. E60, m1359-m1361. 


\section{supporting information}

Acta Cryst. (2005). E61, m2023-m2025 [doi:10.1107/S1600536805028825]

\section{Guanidinium dihydrogenarsenate}

\section{Hazel S. Wilkinson and William T. A. Harrison}

\section{S1. Comment}

The title compound, (I), was prepared as part of on-going studies of hydrogen-bonding interactions in molecular salts (Wilkinson \& Harrison, 2004).

The $\left[\mathrm{H}_{2} \mathrm{AsO}_{4}\right]^{-}$moiety in (I) shows its normal tetrahedral geometry, Fig. 1, about As [mean As- $\mathrm{O}=1.684$ (2) $\AA$ ], with the usual distinction between protonated and un-protonated As-O bond lengths (Wilkinson \& Harrison, 2004); see Table 1. The three $\mathrm{C}-\mathrm{N}$ bond lengths in the propeller-shaped $\left[\mathrm{CH}_{6} \mathrm{~N}_{3}\right]^{+}$entity are similar (Table 1), indicating that the usual model of electron delocalization in this species leading to a $\mathrm{C}-\mathrm{N}$ bond order of 1.33 is applicable here.

As well as Coulombic forces, the component species in (I) interact by means of a network of cation-to-anion $\mathrm{N}-\mathrm{H} \cdots \mathrm{O}$ and anion-to-anion $\mathrm{O}-\mathrm{H} \cdots \mathrm{O}$ H-bonds, as detailed in Table 2. A 11 the guanidinium- $\mathrm{H}$ atoms participate in hydrogen bonds (one of which, via $\mathrm{H} 8$, is bifurcated with notably longer $\mathrm{H} \cdots \mathrm{O}$ separations than the others), such that the $\left[\mathrm{CH}_{6} \mathrm{~N}_{3}\right]^{+}$ moiety makes $\mathrm{N}-\mathrm{H} \cdots \mathrm{O}$ links to five adjacent $\left[\mathrm{H}_{2} \mathrm{AsO}_{4}\right]^{-}$tetrahedra as shown in Fig. 2. For the simple $\mathrm{N}-\mathrm{H} \cdots \mathrm{O}$ bonds, the mean $\mathrm{H} \cdots \mathrm{O}$ distance $=2.18 \AA$, mean $\mathrm{N} \cdots \mathrm{O}=2.951$ (3) $\AA$, and the mean $\mathrm{N}-\mathrm{H} \cdots \mathrm{O}$ angle is $150^{\circ}$.

The $\left[\mathrm{H}_{2} \mathrm{AsO}_{4}\right]^{-}$units are linked into infinte sheets (Fig. 3) by way of the $\mathrm{O}-\mathrm{H} \cdots \mathrm{O}$ hydrogen bonds. The $\mathrm{O} 3-\mathrm{H} 1 \cdots \mathrm{O} 1^{\mathrm{i}}$ interaction (see Table 2 for symmetry codes) results in inversion-symmetry-generated dimeric pairs of $\left[\mathrm{H}_{2} \mathrm{AsO}_{4}\right]^{-}$ tetrahedra linked by a double (i.e. $\mathrm{O}-\mathrm{H} \cdots \mathrm{O}+\mathrm{O} \cdots \mathrm{H}-\mathrm{O}$ ) hydrogen bond. The $\mathrm{O} 4-\mathrm{H} 2 \cdots \mathrm{O} 2{ }^{\text {ii }}$ bond links the dimers into an infinte sheet (Fig. 3) propagating in (100). The As $\cdots A s^{\mathrm{i}}$ and As $\cdots A s^{\mathrm{ii}}$ separations are 4.0148 (3) and 5.0190 (3) $\AA$, respectively. If the topological connectivity of the As atoms is considered, a $6^{3}$ sheet ( $\left.\mathrm{O}^{\prime} \mathrm{Keeffe} \& \mathrm{Hyde}, 1996\right)$ arises, i.e. every As node participates in three polyhedral six-ring loops.

The unit-cell packing for (I) (Fig. 4) results in alternating organic and inorganic layers with respect to the $a$ axis direction. The structure of (I) is distinct from other ammonium hydrogenarsenate salts where isolated pairs of tetrahedra (Todd \& Harrison, 2005) or various kinds of polymeric chains (Wilkinson \& Harrison, 2004) occur.

\section{S2. Experimental}

An aqueous guanidine solution $(0.5 \mathrm{M}, 10 \mathrm{ml})$ was added to an $\mathrm{H}_{3} \mathrm{AsO}_{4}$ solution $(0.5 \mathrm{M}, 10 \mathrm{ml})$ to give a clear solution. A mass of chunks and blocks of (I) grew as the water evaporated over the course of a few days.

\section{S3. Refinement}

The O-bound $\mathrm{H}$ atoms were found in difference maps and fixed in these positions with $U_{\text {iso }}(\mathrm{H})=1.2 U_{\text {eq }}(\mathrm{O})$. The N-bound $\mathrm{H}$ atoms were included in the riding model approximation, with $\mathrm{N}-\mathrm{H}=0.86 \AA$ and with $U_{\text {iso }}(\mathrm{H})=1.2 U_{\text {eq }}(\mathrm{N})$. 


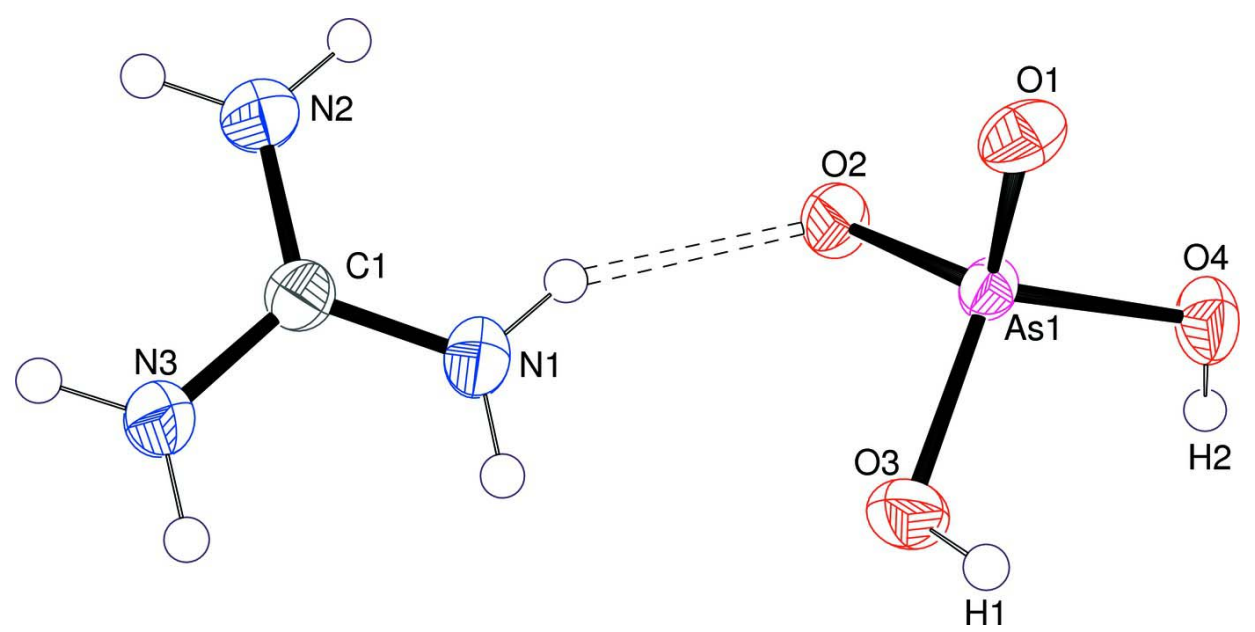

\section{Figure 1}

Asymmetric unit of (I) (50\% displacement ellipsoids). The hydrogen bond is indicated by a dashed line.

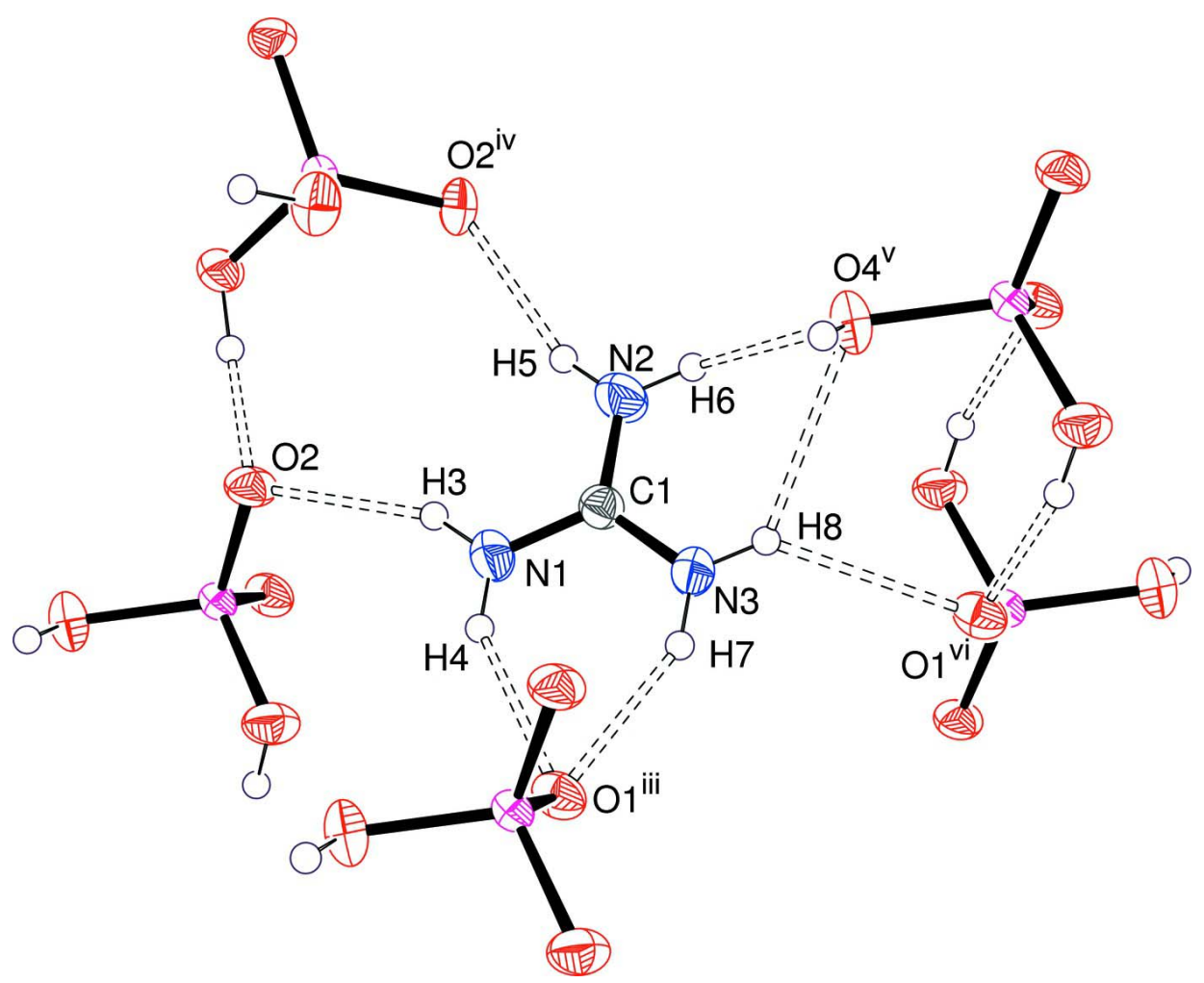

Figure 2

Detail of the cation-to-anion $\mathrm{N}-\mathrm{H} \cdots \mathrm{O}$ links (dashed lines) in (I). Symmetry codes are as in Table 2. 


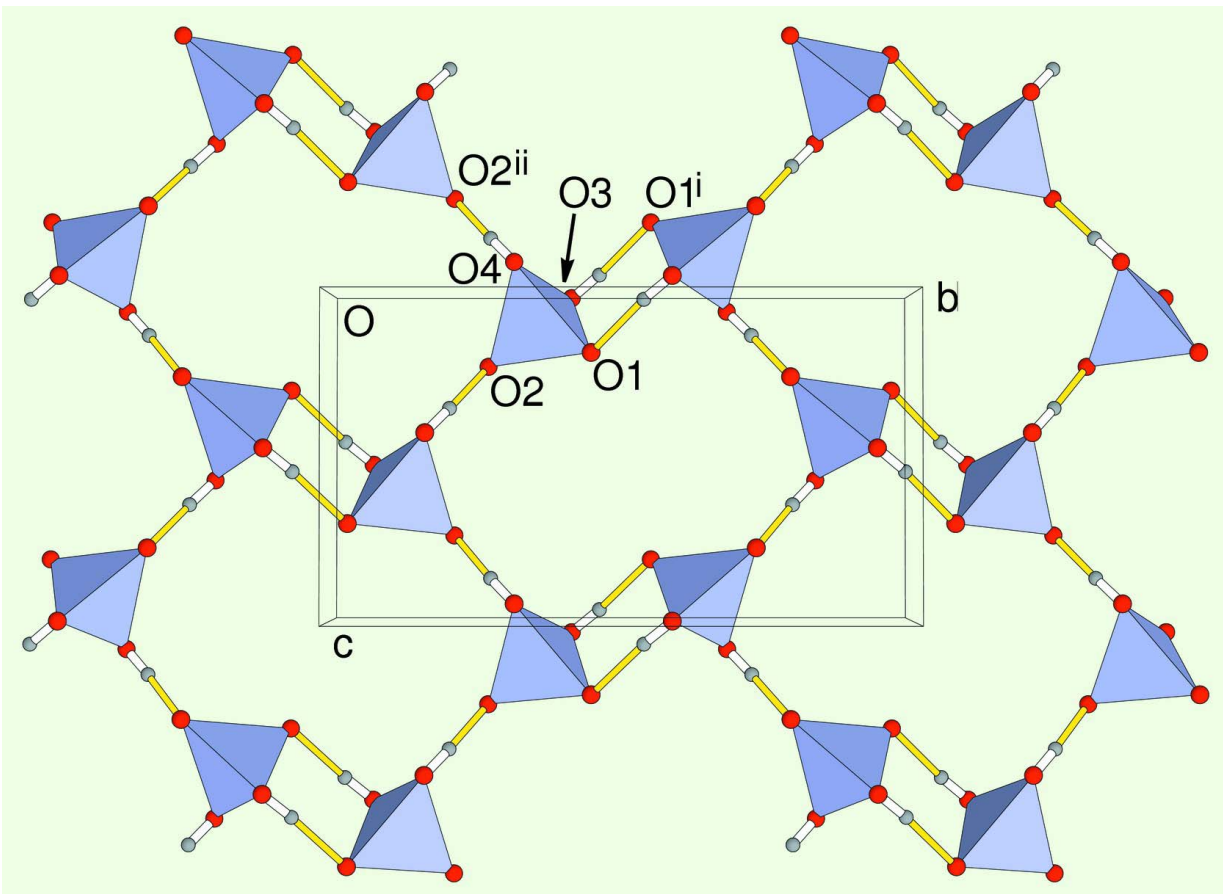

\section{Figure 3}

Detail of a part of a (100) hydrogen-bonded sheet of $\left[\mathrm{H}_{2} \mathrm{AsO}_{4}\right]^{-}$groups in (I), in polyhedral representation. Symmetry codes are as in Table 2. 


\section{supporting information}

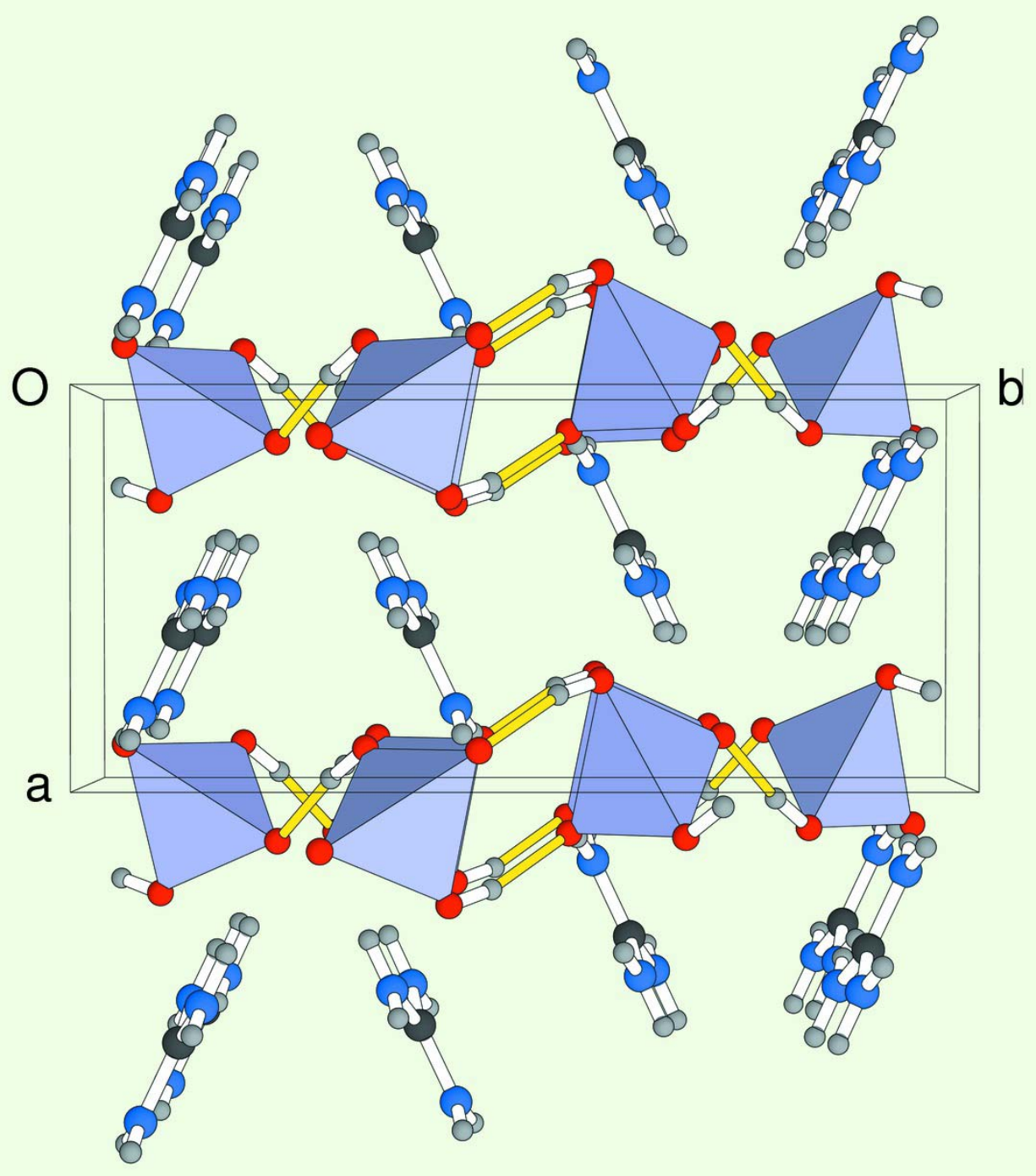

\section{Figure 4}

Unit-cell packing in (I), projected down [001], showing the (100) dihydrogenarsenate layers mediated by guanidinium cations. The $\mathrm{N}-\mathrm{H} \cdots \mathrm{O}$ hydrogen bonds are not shown.

\section{(I)}

\section{Crystal data}

$\left(\mathrm{CH}_{6} \mathrm{~N}_{3}\right)\left[\mathrm{H}_{2} \mathrm{AsO}_{4}\right]$

$M_{r}=201.02$

Monoclinic, $P 2_{1} / c$

Hall symbol: -P 2ybc

$a=6.1571(3) \AA$

$b=13.7052(6) \AA$

$c=7.7208(3) \AA$

$\beta=91.715(1)^{\circ}$

$V=651.22(5) \AA^{3}$

$Z=4$
$F(000)=400$

$D_{\mathrm{x}}=2.050 \mathrm{Mg} \mathrm{m}^{-3}$

Mo $K \alpha$ radiation, $\lambda=0.71073 \AA$

Cell parameters from 4819 reflections

$\theta=3.0-32.5^{\circ}$

$\mu=5.18 \mathrm{~mm}^{-1}$

$T=295 \mathrm{~K}$

Block, colourless

$0.49 \times 0.29 \times 0.13 \mathrm{~mm}$ 


\section{Data collection}

Bruker SMART1000 CCD

diffractometer

Radiation source: fine-focus sealed tube

Graphite monochromator

$\omega$ scans

Absorption correction: multi-scan

(SADABS; Bruker, 1999)

$T_{\min }=0.172, T_{\max }=0.510$

\section{Refinement}

Refinement on $F^{2}$

Least-squares matrix: full

$R\left[F^{2}>2 \sigma\left(F^{2}\right)\right]=0.021$

$w R\left(F^{2}\right)=0.058$

$S=1.06$

2353 reflections

83 parameters

0 restraints

Primary atom site location: structure-invariant direct methods

Secondary atom site location: difference Fourier map

\section{Special details}

Geometry. All e.s.d.'s (except the e.s.d. in the dihedral angle between two 1.s. planes) are estimated using the full covariance matrix. The cell e.s.d.'s are taken into account individually in the estimation of e.s.d.'s in distances, angles and torsion angles; correlations between e.s.d.'s in cell parameters are only used when they are defined by crystal symmetry. An approximate (isotropic) treatment of cell e.s.d.'s is used for estimating e.s.d.'s involving l.s. planes.

Refinement. Refinement of $F^{2}$ against ALL reflections. The weighted $R$-factor $w R$ and goodness of fit $S$ are based on $F^{2}$, conventional $R$-factors $R$ are based on $F$, with $F$ set to zero for negative $F^{2}$. The threshold expression of $F^{2}>\sigma\left(F^{2}\right)$ is used only for calculating $R$-factors $(\mathrm{gt})$ etc. and is not relevant to the choice of reflections for refinement. $R$-factors based on $F^{2}$ are statistically about twice as large as those based on $F$, and $R$-factors based on ALL data will be even larger.

Fractional atomic coordinates and isotropic or equivalent isotropic displacement parameters $\left(\AA^{2}\right)$

\begin{tabular}{lllll}
\hline & $x$ & $y$ & $z$ & $U_{\text {iso }} * / U_{\text {eq }}$ \\
\hline As1 & $0.04340(2)$ & $0.367017(10)$ & $0.104376(16)$ & $0.02035(6)$ \\
O1 & $-0.10944(19)$ & $0.45045(8)$ & $0.19597(13)$ & $0.0295(2)$ \\
O2 & $0.13284(18)$ & $0.27840(8)$ & $0.23321(14)$ & $0.0304(2)$ \\
O3 & $0.27494(18)$ & $0.41686(9)$ & $0.02378(15)$ & $0.0343(3)$ \\
H1 & 0.2406 & 0.4633 & -0.0444 & $0.041^{*}$ \\
O4 & $-0.10648(19)$ & $0.32367(9)$ & $-0.06967(14)$ & $0.0324(2)$ \\
H2 & -0.0288 & 0.2840 & -0.1419 & $0.039 *$ \\
C1 & $0.6107(3)$ & $0.37973(10)$ & $0.5461(2)$ & $0.0256(3)$ \\
N1 & $0.5194(3)$ & $0.36882(10)$ & $0.39137(19)$ & $0.0347(3)$ \\
H3 & 0.3930 & 0.3424 & 0.3803 & $0.042^{*}$ \\
H4 & 0.5860 & 0.3881 & 0.3012 & $0.042^{*}$ \\
N2 & $0.5110(3)$ & $0.35063(13)$ & $0.6865(2)$ & $0.0418(4)$ \\
H5 & 0.3846 & 0.3241 & 0.6772 & $0.050^{*}$ \\
H6 & 0.5727 & 0.3582 & 0.7870 & $0.050^{*}$ \\
N3 & $0.8045(2)$ & $0.42143(11)$ & $0.55908(18)$ & $0.0337(3)$
\end{tabular}

7302 measured reflections

2353 independent reflections

2068 reflections with $I>2 \sigma(I)$

$\theta_{\max }=32.5^{\circ}, \theta_{\min }=3.0^{\circ}$

$h=-9 \rightarrow 9$

$-18 \rightarrow 20$

Hydrogen site location: inferred from

$\mathrm{H}$-atom parameters constrained

$w=1 /\left[\sigma^{2}\left(F_{\mathrm{o}}{ }^{2}\right)+(0.0338 P)^{2}+0.0391 P\right]$

where $P=\left(F_{\mathrm{o}}^{2}+2 F_{\mathrm{c}}^{2}\right) / 3$

( $\sigma)_{\max }<0.001$

$\Delta \rho_{\min }=-0.62$ e $\AA^{-3}$

Extinction correction: SHELXL,

$\mathrm{Fc}^{*}=\mathrm{kFc}\left[1+0.001 \mathrm{xFc}^{2} \lambda^{3} / \sin (2 \theta)\right]^{-1 / 4}$

Extinction coefficient: 0.0145 (12)
$R_{\text {int }}=0.027$ neighbouring sites 
supporting information

\begin{tabular}{lllll}
$\mathrm{H} 7$ & 0.8678 & 0.4407 & 0.4674 & $0.040^{*}$ \\
$\mathrm{H} 8$ & 0.8670 & 0.4292 & 0.6592 & $0.040^{*}$ \\
\hline
\end{tabular}

Atomic displacement parameters $\left(\AA^{2}\right)$

\begin{tabular}{lllllll}
\hline & $U^{11}$ & $U^{22}$ & $U^{33}$ & $U^{12}$ & $U^{13}$ & $U^{23}$ \\
\hline As1 & $0.02193(8)$ & $0.02172(9)$ & $0.01733(8)$ & $0.00041(5)$ & $-0.00081(5)$ & $0.00190(4)$ \\
O1 & $0.0377(6)$ & $0.0279(5)$ & $0.0232(5)$ & $0.0053(5)$ & $0.0067(4)$ & $-0.0013(4)$ \\
O2 & $0.0293(5)$ & $0.0311(6)$ & $0.0305(5)$ & $0.0011(4)$ & $-0.0031(4)$ & $0.0125(4)$ \\
O3 & $0.0268(5)$ & $0.0405(7)$ & $0.0358(6)$ & $-0.0019(5)$ & $0.0041(4)$ & $0.0125(5)$ \\
O4 & $0.0304(5)$ & $0.0377(7)$ & $0.0287(5)$ & $0.0057(5)$ & $-0.0080(4)$ & $-0.0100(5)$ \\
C1 & $0.0269(7)$ & $0.0251(7)$ & $0.0247(6)$ & $0.0004(5)$ & $0.0002(5)$ & $0.0008(5)$ \\
N1 & $0.0322(7)$ & $0.0448(9)$ & $0.0269(7)$ & $-0.0067(6)$ & $-0.0055(5)$ & $0.0017(5)$ \\
N2 & $0.0361(8)$ & $0.0623(10)$ & $0.0272(7)$ & $-0.0125(7)$ & $0.0018(6)$ & $0.0078(7)$ \\
N3 & $0.0327(7)$ & $0.0415(8)$ & $0.0266(6)$ & $-0.0126(6)$ & $-0.0011(5)$ & $-0.0010(5)$ \\
\hline
\end{tabular}

Geometric parameters $\left(\AA,{ }^{\circ}\right)$

\begin{tabular}{|c|c|c|c|}
\hline As1-O1 & $1.6532(11)$ & $\mathrm{C} 1-\mathrm{N} 3$ & $1.324(2)$ \\
\hline $\mathrm{As} 1-\mathrm{O} 2$ & $1.6538(10)$ & $\mathrm{N} 1-\mathrm{H} 3$ & 0.8600 \\
\hline $\mathrm{As} 1-\mathrm{O} 4$ & $1.7135(10)$ & $\mathrm{N} 1-\mathrm{H} 4$ & 0.8600 \\
\hline As $1-\mathrm{O} 3$ & $1.7144(11)$ & $\mathrm{N} 2-\mathrm{H} 5$ & 0.8600 \\
\hline $\mathrm{O} 3-\mathrm{H} 1$ & 0.8491 & $\mathrm{~N} 2-\mathrm{H} 6$ & 0.8600 \\
\hline $\mathrm{O} 4-\mathrm{H} 2$ & 0.9226 & N3- $\mathrm{H} 7$ & 0.8600 \\
\hline $\mathrm{C} 1-\mathrm{N} 1$ & $1.314(2)$ & $\mathrm{N} 3-\mathrm{H} 8$ & 0.8600 \\
\hline $\mathrm{C} 1-\mathrm{N} 2$ & $1.323(2)$ & & \\
\hline $\mathrm{O} 1-\mathrm{As} 1-\mathrm{O} 2$ & $115.74(6)$ & $\mathrm{N} 2-\mathrm{C} 1-\mathrm{N} 3$ & $120.48(16)$ \\
\hline $\mathrm{O} 1-\mathrm{As} 1-\mathrm{O} 4$ & $105.97(6)$ & $\mathrm{C} 1-\mathrm{N} 1-\mathrm{H} 3$ & 120.0 \\
\hline $\mathrm{O} 2-\mathrm{As} 1-\mathrm{O} 4$ & $112.26(6)$ & $\mathrm{C} 1-\mathrm{N} 1-\mathrm{H} 4$ & 120.0 \\
\hline $\mathrm{O} 1-\mathrm{As} 1-\mathrm{O} 3$ & $111.71(6)$ & $\mathrm{H} 3-\mathrm{N} 1-\mathrm{H} 4$ & 120.0 \\
\hline $\mathrm{O} 2-\mathrm{As} 1-\mathrm{O} 3$ & $104.21(6)$ & $\mathrm{C} 1-\mathrm{N} 2-\mathrm{H} 5$ & 120.0 \\
\hline $\mathrm{O} 4-\mathrm{As} 1-\mathrm{O} 3$ & $106.72(6)$ & $\mathrm{C} 1-\mathrm{N} 2-\mathrm{H} 6$ & 120.0 \\
\hline As1-O3-H1 & 109.2 & $\mathrm{H} 5-\mathrm{N} 2-\mathrm{H} 6$ & 120.0 \\
\hline $\mathrm{As} 1-\mathrm{O} 4-\mathrm{H} 2$ & 113.7 & $\mathrm{C} 1-\mathrm{N} 3-\mathrm{H} 7$ & 120.0 \\
\hline $\mathrm{N} 1-\mathrm{C} 1-\mathrm{N} 2$ & $120.96(16)$ & $\mathrm{C} 1-\mathrm{N} 3-\mathrm{H} 8$ & 120.0 \\
\hline $\mathrm{N} 1-\mathrm{C} 1-\mathrm{N} 3$ & $118.57(15)$ & $\mathrm{H} 7-\mathrm{N} 3-\mathrm{H} 8$ & 120.0 \\
\hline
\end{tabular}

Hydrogen-bond geometry $\left(\AA,{ }^{o}\right)$

\begin{tabular}{lllll}
\hline$D-\mathrm{H} \cdots A$ & $D-\mathrm{H}$ & $\mathrm{H} \cdots A$ & $D \cdots A$ & $D-\mathrm{H} \cdots A$ \\
\hline $\mathrm{O} 3-\mathrm{H} 1 \cdots \mathrm{O} 1^{\mathrm{i}}$ & 0.85 & 1.83 & $2.6684(16)$ & 167 \\
$\mathrm{O} 4-\mathrm{H} 2 \cdots \mathrm{O} 2^{\mathrm{ii}}$ & 0.92 & 1.65 & $2.5648(16)$ & 174 \\
$\mathrm{~N} 1-\mathrm{H} 3 \cdots \mathrm{O} 2$ & 0.86 & 2.13 & $2.9185(18)$ & 153 \\
$\mathrm{~N} 1-\mathrm{H} 4 \cdots \mathrm{O} 1^{\mathrm{iii}}$ & 0.86 & 2.24 & $2.993(2)$ & 147 \\
$\mathrm{~N} 2-\mathrm{H} 5 \cdots 2^{\mathrm{iv}}$ & 0.86 & 2.15 & $2.955(2)$ & 157 \\
$\mathrm{~N} 2-\mathrm{H} 6 \cdots 4^{v}$ & 0.86 & 2.28 & $2.994(2)$ & 140 \\
$\mathrm{~N} 3-\mathrm{H} 7 \cdots \mathrm{O} 1^{\mathrm{iii}}$ & 0.86 & 2.11 & $2.8962(17)$ & 152
\end{tabular}




\section{supporting information}

$\begin{array}{lllll}\mathrm{N} 3-\mathrm{H} 8 \cdots \mathrm{O} 1^{\mathrm{vi}} & 0.86 & 2.47 & 3.1582(18) & 138 \\ \mathrm{~N} 3-\mathrm{H} 8 \cdots \mathrm{O}^{\mathrm{v}} & 0.86 & 2.55 & 3.1965(18) & 133\end{array}$

Symmetry codes: (i) $-x,-y+1,-z$; (ii) $x,-y+1 / 2, z-1 / 2$; (iii) $x+1, y, z$; (iv) $x,-y+1 / 2, z+1 / 2$; (v) $x+1, y, z+1$; (vi) $-x+1,-y+1,-z+1$. 